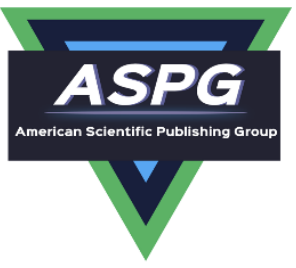

\title{
A Single Valued neutrosophic Inventory Model with Neutrosophic Random Variable
}

\author{
M. Mullai1*, K. Sangeetha2, R. Surya3, G. Madhan kumar4, R. Jeyabalan5, S. Broumi6 \\ Department of Mathematics, Alagappa University, Karaikudia. \\ Email: mullaim@alagappauniversity.ac.in, sangeekannan07@gmail.com, suryarrrm@gmail.com, \\ madhan001kumar@gmail.com, jeyram84@gmail.com, broumisaid78@gmail.com
}

\begin{abstract}
This paper presents the problematic period of neutrosophic inventory in an inaccurate and unsafe mixed environment. The purpose of this paper is to present demand as a neutrosophic random variable. For this model, a new method is developed for determining the optimal sequence size in the presence of neutrosophic random variables. Where to get optimality by gradually expressing the average value of integration. The newsvendor problem is used to describe the proposed model.
\end{abstract}

Keywords: Neutrosophic set, Neutrosophic random variable, Triangle neutrosophic numbers, single period neutrosophic inventory

\section{Introduction}

A single-period inventory (Buffa E.S. and Sarin R.K. 1987) is one of those elementary models in which only a single procurement is being made. There is a wide application of this model on production management system, like stocking seasonal items (Christmas trees, woolen materials),perishable goods, spare parts, etc. To develop the methodology of this model, we consider the well-known newsboy problem, in which the decision-maker wants to know the optimal number of newspapers to be purchased daily to maximize his expected profit. In a real situation, the daily demand of the newspapers may vary day to day. Either due to lack of historical data or abundance of information it is worthwhile to consider a distribution for demand. Recently some researchers considered the demand as a fuzzy number only (Kao C. and Hsu W.K. 2002). In (Ishii H. and Konno T. 1998) the newsboy problem has been redefined considering shortage cost as fuzzy number and demand as random variable. However, no attempt is made to define the demand in mixed environment, where fuzziness and randomness both appears simultaneously. Thus, we consider the demand as a fuzzy random variable involving imprecise probabilities since the probability of a fuzzy event is a fuzzy number (Chakraborthy D. 2002).

The concept of fuzzy random variable and its fuzzy expectation has been presented by (Kwakernaak H. 1978) and later by Puri and Ralescue (Puri M.L. andRalescu D.A. 1986). Further, recently the notation of a fuzzy random variable has also been considered in (Feng Y., Hu L. and Shu H. 2001). In (Smarandache F. 1998) proposed concept of neutrosophic set which is generalization of classical set, fuzzy set, intuitionistic fuzzy set and so on. In the neutrosophic set, for an element $\mathrm{x}$ of the universe, the membership functions independently indicates the truth- 
membership degree, indeterminacy-membership degree, and false-membership degree of the element $\mathrm{x}$ belonging to the neutrosophic set. Also, fuzzy, intuitionistic and neutrosophic models have been studied by (Wang $\mathrm{H}$, Smarandache F, Y, Zhang Q 2010). In a multiple-attribute decision-making problem the decision makers need to rank the given alternatives and the ranking of alternatives with neutrosophic numbers is many is many difficult because neutrosophic numbers are not ranked by ordinary methods as real numbers. However it is possible with score functions, aggregation operation, distance measure and so on. In section 2 of this paper, the neutrosophic random variable and its neutrosophic expectation are defined, a brief overview of the integration of graded mean representation of triangular neutrosophic number discussed later. Next, in section 3 a single-valued neutrosophic inventory problem of neutrosophic random variable demand is formulated and methodology is developed. Section 4 handles the numerical example of the proposed model.

\section{Preliminaries}

In this section, the basic definitions involving neutrosophic set, single valued neutrosophic sets and triangularneutrosophic number are outlined.

Definition 1. (Smarandache F. 1998)Let $E$ be a universe. A neutrosophic set $A$ over $E$ is defined by, $A=\left\{\left(x_{v}\left(T_{A}(x), I_{A}(x), F_{A}(x)\right)\right\}: x \in E\right\}$ where $T_{A}(x), I_{A}(x)$ and $F_{A}(x)$ are called truth-membership function, indeterminacy-membership function and falsity-membership function respectively. They are respectively defined by, $\left.\mathrm{T}_{\mathrm{A}}: \mathrm{E}-\right]^{-} 0,1^{+}\left[, \mathrm{I}_{\mathrm{A}}: \mathrm{E} \rightarrow\right]^{-} 0,1^{+}\left[, \mathrm{F}_{\mathrm{A}}: \mathrm{E} \rightarrow\right]^{-} 0,1^{+}\left[\right.$such that $0^{-} \leq \mathrm{T}_{\mathrm{A}}(\mathrm{x})+\mathrm{I}_{\mathrm{A}}(\mathrm{x})+\mathrm{F}_{\mathrm{A}}(\mathrm{x}) \leq 3^{+}$.

Definition 2. (Wang H, Smarandache F. Zhang Y.Q 2010)Let E be a universe. A single valued neutrosophic set (SVN-set) over E, but the truth-membership function, indeterminacy-membership function and falsity-membership function are respectively defined by $\left.\mathrm{T}_{\mathrm{A}}: \mathrm{E}-\right]^{-} 0,1^{+}\left[, \mathrm{I}_{\mathrm{A}}: \mathrm{E} \rightarrow\right]^{-} 0,1^{+}\left[, \mathrm{F}_{\mathrm{A}}: \mathrm{E} \rightarrow\right]^{-} 0,1^{+}[$such that $0 \leq \mathrm{T}_{\mathrm{A}}(\mathrm{x}), \mathrm{I}_{\mathrm{A}}(\mathrm{x}), \mathrm{F}_{\mathrm{A}}(\mathrm{x}) \leq 3$.

Definition 3. (Subas Y. 2015)Let $w_{\mathrm{a}}, \mathrm{u}_{\mathrm{a}}, \mathrm{y}_{\mathrm{a}} \in[0,1]$ be any real numbers, $\mathrm{a}_{\mathrm{i}}, \mathrm{b}_{\mathrm{i}}, \mathrm{c}_{\mathrm{i}} \in \mathrm{R}$ and $\mathrm{a}_{\mathrm{i}} \leq \mathrm{b}_{\mathrm{i}} \leq \mathrm{c}_{\mathrm{i}}(\mathrm{i}=1,2,3)$. Then single valued neutrosophic number ( $\mathrm{SVN}$-number) $\tilde{\mathrm{a}}=\left\langle\left(\left(\mathrm{a}_{1}, \mathrm{~b}_{1}, \mathrm{c}_{1}\right), \mathrm{w}_{\mathrm{a}}\right),\left(\left(\mathrm{a}_{2}, \mathrm{~b}_{2}, \mathrm{c}_{2}\right), \mathrm{u}_{\mathrm{a}}\right),\left(\left(\mathrm{a}_{\mathrm{a}}, \mathrm{b}_{\mathrm{a}}, \mathrm{c}_{\mathrm{a}}\right), \mathrm{y}_{\mathrm{a}}\right)\right\rangle$, is a special neutrosophic set on the set of real number $\mathrm{R}$, whose truth-membership function $\mu_{\mathrm{a}}$, indeterminacy-membership function $v_{\mathrm{a}}$ and falsity-membership function $\lambda_{\mathrm{a}}$ are respectively defined by $\mu_{\mathrm{a}}: \mathrm{R} \rightarrow\left[0, \mathrm{w}_{\mathrm{a}}\right], \mathrm{v}_{\mathrm{a}} \mathrm{R} \rightarrow\left[\mathrm{u}_{\mathrm{d}}, 1\right], \lambda_{\mathrm{a}} \mathrm{z}, \mathrm{R} \rightarrow\left[\mathrm{y}_{\mathrm{a}}, 1\right]$.

Definition 4. (Subas Y. 2015)A single valued triangular neutrosopic number $\left\langle\left(a_{v}, b_{s} c\right) ; w_{\mathbb{E}}, u_{\mathbb{E}} s y_{\mathbb{E}}\right\rangle$, is a special neutrosopic set on the real number set $R$, whose truth-membership, indeterminacy -membership and falsitymembership are given as follows;

$$
\begin{aligned}
& \mu_{\mathbb{Q}}(x)=\left\{\begin{array}{lc}
(x-a) w_{\mathbb{Q}} /(b-a), & (a \leq x<b) \\
(c-x) w_{\mathbb{Q}} /(c-b), & (b \leq x \leq c) \\
0, & \text { otherwise }
\end{array}\right. \\
& v_{a}(x)=\left\{\begin{array}{cc}
\left(b-x+u_{\mathbb{Q}}(x-a)\right) /(b-a), & (a \leq x<b) \\
\left(x-b+u_{\mathbb{Q}}(c-x)\right) /(c-b), & (b \leq x \leq c) \\
0, & \text { otherwise }
\end{array}\right. \\
& \lambda_{a}(x)= \begin{cases}\frac{\left(b-x+y_{a}(x-a)\right)}{(b-a)}, & (a \leq x<b) \\
\frac{\left(x-b+y_{a}(c-x)\right)}{(c-b)}, & (b \leq x \leq c) \\
0, & \text { otherwise }\end{cases}
\end{aligned}
$$


Definition 5. (Deli I, Subas y) Let $\mathrm{a}=\left\langle\left(\left(\mathrm{a}_{1}, \mathrm{~b}_{1}, \mathrm{c}_{1}\right), \mathrm{w}_{\mathrm{a}}\right),\left(\left(\mathrm{a}_{2}, \mathrm{~b}_{2}, \mathrm{c}_{2}\right), \mathrm{u}_{\mathrm{a}}\right),\left(\left(\mathrm{a}_{\mathrm{g}}, \mathrm{b}_{\mathrm{a}}, \mathrm{c}_{\mathrm{a}}\right), \mathrm{y}_{\mathrm{a}}\right)\right\rangle$ be a SVN - Number. Then $\langle\alpha, \beta, \gamma\rangle$ - cut set of theSVN - Numberã, denoted by $\tilde{a}_{\{a, \beta,}$ is defined as ,$\tilde{Q}_{\alpha_{\alpha}, \beta \eta}=\left\{x / \mu_{a}(x) \geq \alpha, v_{a}(x) \leq \beta, \lambda_{a}(x) \leq \gamma, x \in R\right\}$, which satisfies the conditions as follows:

$0 \leq \alpha \leq w_{\mathrm{a}}, \mathrm{u}_{\mathrm{a}} \leq \beta \leq 1, \mathrm{y}_{\mathrm{a}} \leq \gamma \leq 1$ and $0 \leq \alpha+\gamma+\beta \leq 3$.

Clearly, any $\langle\alpha, \beta, \gamma\rangle$-cut set $\tilde{a}_{\left\{\alpha_{\beta}, \beta,\right.}$ of a SVN - Numberã is a crisp subset of a real number set $R$.

Definition 6.(Deli I, Subas y) Let ã $=\left\langle\left(\left(\mathrm{a}_{1}, \mathrm{~b}_{1}, \mathrm{c}_{1}\right), \mathrm{w}_{\mathrm{a}}\right),\left(\left(\mathrm{a}_{2}, \mathrm{~b}_{2}, \mathrm{c}_{2}\right), \mathrm{u}_{\mathrm{a}}\right),\left(\left(\mathrm{a}_{\mathrm{a}}, \mathrm{b}_{\mathrm{a}}, \mathrm{c}_{\mathrm{a}}\right), \mathrm{y}_{\mathrm{a}}\right)\right)$ be a SVN - Number. Then $\alpha$ - cut set of theSVN - Numberã, denoted by $\widetilde{a}_{\alpha}$ is defined as , $\tilde{a}_{\alpha}=\left\{x / \mu_{\alpha}(x) \geq \alpha_{s} x \in R\right\}$, where $\alpha \in\left[0, w_{\mathfrak{a}}\right]$.

Clearly, any $\alpha$-cut set of a SVN - Numberã is a crisp subset of a real number set R. Also any $\alpha$-cut set of a SVNNumber ãfor truth-membership function is a closed interval, denoted by $\tilde{a}_{\alpha}=\left[L_{a}(\alpha), R_{a}(\alpha)\right]$.

Definition 7.(Deli I, Subas y) Let $\mathbf{a}=\left\langle\left(\left(\mathrm{a}_{1}, \mathrm{~b}_{1}, \mathrm{c}_{1}\right), \mathrm{w}_{\mathrm{a}}\right),\left(\left(\mathrm{a}_{2}, \mathrm{~b}_{2}, \mathrm{c}_{2}\right), \mathrm{u}_{\mathrm{a}}\right),\left(\left(\mathrm{a}_{\mathrm{a}}, \mathrm{b}_{\mathrm{a}}, \mathrm{c}_{\mathrm{a}}\right), \mathrm{y}_{\mathrm{a}}\right)\right)$ be a SVN - Number. Then $\beta$ - cut set of theSVN - Numberã, denoted by $\tilde{a}^{\beta}$ is defined as $\tilde{a}^{\beta}=\left\{x / v_{a}(x) \leq \beta, x \in R\right\}$, where $\beta \in\left[u_{a}, 1\right]$.

Clearly, any $\beta$-cut set of a SVN - Numberã is a crisp subset of a real number set $R_{n}$ Also any $\beta$-cut set of a SVN-Number ãfor indeterminacy-membership function is a closed interval, denoted by $\tilde{a}^{\beta}=\left[L^{v}(\beta), R_{a}^{\prime}(\beta)\right]$.

Definition 8.(Deli I, Subas y) Let a $=\left\langle\left(\left(a_{1}, b_{1}, c_{1}\right), w_{2}\right),\left(\left(a_{2}, b_{2}, c_{2}\right), u_{a}\right),\left(\left(a_{2}, b_{2}, c_{2}\right), y_{a}\right)\right\rangle$ be a SVN - Number. Then $\gamma$ - cut set of theSVN - Numberã, denoted by $\gamma_{\mathrm{a}}$ is defined as,$\gamma_{\mathrm{a}}=\left\{\mathrm{x} / \lambda_{\mathrm{a}}(\mathrm{x}) \leq \gamma, \mathrm{x} \in \mathrm{R}\right\}$, where $\gamma \in\left[\mathrm{y}_{\mathrm{a}}, 1\right]$.

Clearly, any $\gamma$-cut set of a SVN - Numberã is a crisp subset of a real number set R. Also any $\gamma$-cut set of a SVNNumber ãfor indeterminacy-membership function is a closed interval, denoted by $\gamma_{\mathrm{a}}=\left[\mathrm{L}_{\mathrm{a}}^{n p}(\gamma), \mathrm{R}_{\mathrm{Q}}^{n}(\gamma)\right]$.

\section{Cuts and neutrosophicgraded mean integration:}

$\alpha, \beta$ and $\gamma$-cuts, expectation of neutrosophic random variable and neutrosophic graded mean are introduced in this section.

Definition 1. Let $F^{\mathbb{N}}$ be the set of all neutrosophic numbers. The $\alpha$-cut, $\beta$-cut and $\gamma$-cut of $w_{a^{N}}$, $u_{a} \mathbb{N}_{y}$ andy $a^{\mathbb{N}}$ in $F^{\mathbb{N}}$ is closed interval of any $\alpha, \beta, \gamma \in \mathrm{F}^{\mathbb{N}}$. The addition and scalar multiplication on $\mathrm{F}^{\mathbb{N}}$ are defined by the following;

$[a, b]_{\mathbb{E}}^{N}=a_{\mathbb{E}}^{N}+b_{\mathbb{E}}^{N},[\lambda a]_{\mathbb{E}}^{N}=\lambda a_{\mathbb{E}}^{N}, \lambda \in R, \quad a \in[0,1]$

$[a, b]_{\beta}^{N}=a_{\beta}^{N}+b_{\beta}^{N},[\lambda a]_{\beta}^{N}=\lambda o_{\beta}^{N}, \lambda \in R, \quad \beta \in[0,1]_{;}$

$[a, b]_{Y}^{\mathbb{N}}=a_{Y}^{N}+b_{Y}^{N},[\lambda a]_{Y}^{N}=\lambda a_{Y}^{N}, \lambda \in R, y \in[0,1] ; a^{N}, b^{N} \in F^{N}$ and

$[a, b]_{\alpha+\beta+Y}^{N}=a_{\alpha+\beta+Y}^{N}+b_{\alpha+\beta+Y^{s}}^{N}[\lambda a]_{\alpha+\beta+Y}^{N}=\lambda a_{\alpha+\beta+Y^{s}}^{N} \lambda \in R, \alpha+\beta+\gamma \in[0,3]$.

A metric on $F^{\mathbb{N}}$ is defined by,

$d^{N}\left(a^{N}, b^{N}\right)=\frac{1}{2} \int_{0}^{1}\left(\left(\left|a_{⿷}^{-}-b_{a}^{-}\right|\right)^{2}+\left(\left|a_{E}^{+}+b_{\mathbb{E}}^{+}\right|\right)^{2}\right) d^{N} a_{s} \quad \forall a^{N}, b^{N} \in F^{N}$

$d^{N}\left(a^{N}, b^{N}\right)=\frac{1}{2} \int_{0}^{1}\left(\left(\left|a_{\beta}^{-}-b_{\beta}^{-}\right|\right)^{2}+\left(\left|a_{\beta}^{+}+b_{\beta}^{+}\right|\right)^{2}\right) d^{N} \beta, \quad \forall a^{N}, b^{N} \in F^{N}$ 


$$
\begin{aligned}
& d^{N}\left(a^{N}, b^{N}\right)= \frac{1}{2} \int_{0}^{1}\left(\left(\left|a_{Y}^{-}-b_{Y}^{-}\right|\right)^{2}+\left(\left|a_{Y}^{+}+b_{Y}^{+}\right|\right)^{2}\right) d^{N} \gamma, \forall a^{N}, b^{N} \in F^{N} \text { and } \\
& d^{N}\left(\alpha^{N}, b^{N}\right)= \frac{1}{2} \int_{0}^{1}\left(\left(\left|a_{⿷+\beta+Y}^{-}-b_{\alpha+\beta+\gamma}^{-}\right|\right)^{2}+\left(\left|a_{\alpha+\beta+Y}^{+}+b_{\alpha+\beta+\gamma}^{+}\right|\right)^{2}\right) d^{N}(\alpha+\beta+\gamma), \\
& \forall a^{N}, b^{N} \in F^{N},(\alpha+\beta+\gamma) \in[0,3],
\end{aligned}
$$

Where $\mathrm{a}_{\mathbb{\alpha}}^{-}, \mathrm{a}_{\mathbb{a}}^{+}$are lower and upper end point of $\mathrm{a}_{\mathbb{\alpha}}$ and $\left(\mathrm{F}^{\mathbb{N}}, \mathrm{d}^{\mathbb{N}}\right)$ is a complete neutrosophic metric space.

Let $\left(\Omega^{\mathbb{N}}{ }_{r} A^{N}{ }_{s} P^{N}\right)$ be a complete neutrosophic probability space. A neutrosophic random variable (n.r.v) is a borel measurable function $\mathrm{X}^{\mathbb{N}_{s}}\left(\Omega^{\mathrm{N}}{ }_{s} \mathrm{~A}^{\mathrm{N}}{ }_{\mathrm{s}} \mathrm{P}^{\mathrm{N}}\right) \rightarrow\left(\mathrm{F}^{\mathrm{N}}{ }_{\mathrm{s}} \mathrm{d}^{\mathrm{N}}\right)$.

If $\mathrm{X}^{\mathrm{N}}$ is a n.r.v, then $[\mathrm{X}]_{\mathbb{\Omega}}^{\mathbb{N}}=\left[\mathrm{X}_{\mathbb{\alpha}}^{-}, \mathrm{X}_{\mathbb{\Omega}}^{+}\right]^{\mathrm{N}},[\mathrm{X}]_{\beta}^{\mathbb{N}}=\left[\mathrm{X}_{\beta}^{-}, \mathrm{X}_{\beta}^{+}\right]^{\mathbb{N}},[\mathrm{X}]_{\mathrm{Y}}^{\mathrm{N}}=\left[\mathrm{X}_{\mathrm{Y}}^{-}, \mathrm{X}_{\mathrm{Y}}^{+}\right]^{\mathrm{N}}$, and

$[\mathrm{X}]_{\alpha+\beta+Y}^{\mathrm{N}}=\left[\mathrm{X}_{\alpha+\beta+Y_{Y}}^{-}, X_{\alpha+\beta+\gamma}^{+}\right]^{\mathrm{N}}, \alpha+\beta+\gamma \in[0,3]$ is neutrosophic random closed interval set and $\mathrm{X}_{\alpha+\beta+Y^{-}}^{-}, X_{\alpha+\beta+Y}^{+}$ are real valued neutrosophic random variables. The expectation of a n.r.v $\mathrm{X}^{\mathrm{N}}$ is defined by

$a_{\alpha+\beta+Y}=[E X]_{\alpha+\beta+Y}^{N}=E^{N}\left[X_{\alpha+\beta+\gamma}\right]=\left[E^{\mathbb{N}}\left(X_{\alpha+\beta+Y}^{-}\right), E^{N}\left(X_{\alpha+\beta+Y}^{+}\right)\right]$

for $\alpha+\beta+\gamma \in[0,3], \alpha, \beta, \gamma \in[0,1]$.

Definition 2. For a neutrosophic random variable

$\mathrm{X}^{\mathbb{N}}=\left\{\left(\mathrm{X}_{\alpha+\beta+\gamma^{\top}}^{-} \mathrm{X}_{\alpha+\beta+\gamma}^{+}\right)^{\mathbb{N}} / 0 \leq \alpha+\beta+\gamma \leq 3\right\}$ the expectation of $\mathrm{X}^{\mathbb{N}}$ is defined by

$E^{\mathbb{N}} X^{\mathbb{N}}=\int X^{\mathbb{N}} d P^{N}=\left\{\int X_{\alpha+\beta+Y}^{-} d P^{N}, \int X_{\alpha+\beta+Y}^{+} d P^{\mathbb{N}} / 0 \leq \alpha+\beta+\gamma \leq 3\right\}$.

If $\mathrm{X}^{\mathbb{N}}$ is a discrete neutrosophic random variable, such that $\mathrm{P}^{\mathbb{N}}\left(\mathrm{X}^{\mathbb{N}}=\mathrm{x}_{\mathrm{i}}^{\mathrm{N}}\right)=\mathrm{p}_{\mathrm{i}}^{\mathrm{N}}, \mathrm{i}=1,2,3, \ldots$, then its neutrosophic expected is given as,

$\mathrm{EX}^{\mathbb{N}}=\sum_{\mathrm{i}=1}^{\infty} \mathrm{x}_{\mathrm{i}}^{\mathrm{N}} \mathrm{p}_{\mathrm{i}}^{\mathrm{N}}$, forEX $\mathrm{X}^{\mathbb{N}} \in \mathrm{F}^{\mathbb{N}}$ and $\left[\mathrm{EX}^{\mathbb{N}}\right]_{0}=\int \mathrm{X}_{0} \mathrm{dP}^{\mathrm{N}}=\left[\mathrm{EX}_{0}^{-}, \mathrm{EX}_{0}^{+}\right]^{\mathbb{N}}$, for

$\alpha+\beta+\gamma=0$.

To achieve computational efficiency the method of discovering a neutrosophic number becomes a graded representation of the average integration.

A generalized neutrosophic number $T_{A}+I_{A}+F_{A}$ is explained as any neutrosophic subset of the real line $R$, whose membership function $\mu_{\mathbb{N}_{\Lambda}+\mathrm{I}_{\Lambda}+\mathrm{F}_{\Lambda}}^{\mathrm{N}}$ (u) satisfies as follows,

i) $\mu_{\mathrm{I}_{A}+\mathrm{I}_{\Lambda}+\mathrm{F}_{\Lambda}}^{\mathbb{N}}$ (u) is continuous neutrosophic mapping from $\mathrm{R}$ to $[0,1]$,

ii) $\mu_{\mathbb{I}_{A}+\mathrm{I}_{\Lambda}+\mathrm{F}_{\Lambda}}^{\mathrm{N}}(\mathrm{u})=0, \infty<\mathrm{u} \leq \underline{\mathrm{a}}$,

iii) $\mu_{\mathrm{L}}^{\mathbb{N}}(\mathrm{u})=\mathrm{L}(\mathrm{u})$ is strictly increasing on $[\underline{\mathrm{a}}, \mathrm{a}]$,

iv) $\mu_{\Gamma_{\Lambda}+I_{\Lambda}+E_{\Lambda}}^{\mathbb{N}}(\mathrm{u})=w, u=a$, 
v) $\mu_{\mathrm{R}}^{\mathrm{N}}(\mathrm{u})=\mathrm{R}(\mathrm{u})$ is strictly decreasing on $\left[\mathrm{a}_{s} \overline{\mathrm{a}}\right]$, where $0<\mathrm{w} \leq 3$ and $\mathrm{a}_{s} \overline{\mathrm{a}}_{s} \underline{\underline{\mathbf{a}}}$ are real numbers.

This type of generalized neutrosophic number is a triangular neutrosophic number, and its denoted by $T_{A}+I_{A}+F_{A}=$ $\left(\mathrm{a}, \overline{\mathrm{a}}_{2}, \mathrm{a}, \mathrm{w}\right) \stackrel{\mathrm{N}}{\mathrm{LR}}$. When $\mathrm{w}=1$, this kind of generalized neutrosophic number is called normal neutrosophic number and its characterization, $\mathrm{T}_{\mathrm{A}}+\mathrm{I}_{\mathrm{A}}+\mathrm{F}_{\mathrm{A}}=\left(\mathrm{a}, \overline{\mathrm{a}}_{2}, \underline{\mathrm{a}}\right)_{\mathrm{LR}}^{\mathrm{N}}$.

The graded mean $\alpha+\beta+\gamma$-level value of $T_{A}+I_{A}+F_{A}=\left(a, \bar{a}_{2}, \underline{a}\right)_{\text {LR }}^{\mathbb{N}}$ is

$(\alpha+\beta+\gamma)[L(\alpha+\beta+\gamma)+R(\alpha+\beta+\gamma)] / 2$. Therefore the graded mean integration characterization of generalized triangular neutrosophic number $T_{A}+I_{A}+F_{A}$ is,

$$
\begin{aligned}
& \mathrm{G}\left(T_{A}+I_{A}+F_{A}\right)=
\end{aligned}
$$

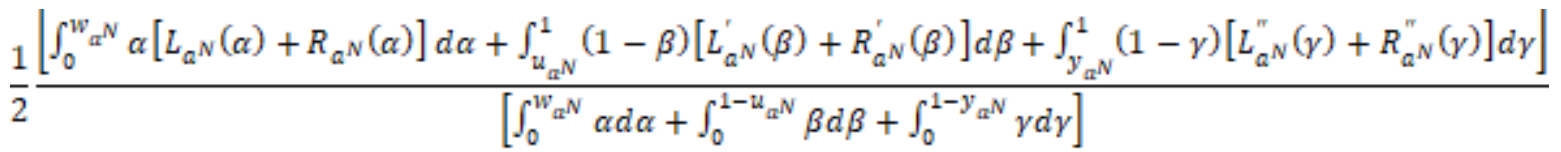

$$
\begin{aligned}
& \int_{0}^{W_{\omega^{N}}^{N}} \alpha\left[L_{a^{N}}(\alpha)+R_{\alpha^{N}}(\alpha)\right] d \alpha=\int_{0}^{W_{\alpha^{N}}}\left[(a+c)+\frac{(2 b-b-c) \alpha}{w_{a^{N}}}\right] \alpha d \alpha \\
& \left\{\text { where }\left[L_{a^{N}}(\alpha)+R_{a^{N}}(\alpha)\right]=\left[\frac{\left(w_{a^{N}}-a\right) a+\alpha b}{w_{a^{N}}}, \frac{\left(w_{a^{N}}-a\right) c+\alpha b}{w_{a^{N}}}\right]\right\} \\
& =\left[\frac{(a+c) \alpha^{2}}{2}+\frac{(2 b-a-c) \alpha^{3}}{3 w_{\alpha^{N}}}\right]_{0}^{w_{a^{N}}} \\
& =\left[\frac{(a+c) w_{a^{N}} N^{2}}{2}+\frac{(2 b-a-c) w_{a^{N}}{ }^{a}}{3 w_{a^{N}}}\right] \\
& =\frac{(3 a+3 c+4 b-2 a-2 c) w_{a^{N}}{ }^{2}}{6} \\
& =\frac{(a+4 b+c) w_{a^{N}}{ }^{2}}{6} \\
& \int_{u_{a^{N}}}^{1}(1-\beta)\left[L_{\alpha^{N}}^{s}(\beta)+R_{\alpha^{N}}^{s}(\beta)\right] d \beta=\int_{u_{a^{N}}}^{1}\left[(a+c)+\frac{(2 b-a-c)(1-\beta)}{1-u_{a^{N}}}\right](1-\beta) d \beta_{x} \\
& \left\{\text { where }\left[L_{a^{N}}^{s}(\beta)+R_{\mathbb{a}^{N}}^{s}(\beta)\right]=\left[\frac{(1-\beta) b+\left(\beta-u_{\alpha^{N}}\right) a}{1-u_{a^{N}}}, \frac{(1-\beta) b+\left(\beta-u_{\alpha^{N}}\right) c}{1-u_{a^{N}}}\right]\right\} \\
& =\left[\frac{(a+c)(1-\beta)^{2}}{2}+\frac{(2 b-a-c)(1-\beta)^{3}}{3\left(1-u_{a^{N}}\right)}\right]_{u_{a^{N}}}^{1}
\end{aligned}
$$




$$
\begin{aligned}
& =\left[\frac{(a+c)\left(1-u_{\left.a^{N}\right)^{2}}\right.}{2}+\frac{(2 b-a-c)\left(1-u_{\left.a^{N}\right)^{3}}\right.}{3\left(1-u_{a^{N}}\right)}\right] \\
& =\frac{(3 a+3 c+4 b-2 a-2 c)\left(1-u_{\left.a^{N}\right)^{2}}\right.}{6} \\
& =\frac{(a+4 b+c)\left(1-u_{\left.a^{N}\right)^{2}}\right.}{6}
\end{aligned}
$$

$$
\int_{y_{\mathbb{L}^{N}}}^{1}(1-\gamma)\left[L_{a^{N}}^{s}(\gamma)+R_{a^{N}}^{s}(\gamma)\right] d y=\int_{y_{\mathbb{L}^{N}}}^{1}\left[(a+c)+\frac{(2 b-a-c)(1-\gamma)}{1-y_{a^{N}}}\right](1-\gamma) d \gamma_{x}
$$

$$
\left\{\text { where }\left[L_{a^{N}}^{s}(\gamma)+R_{a^{N}}^{s}(\gamma)\right]=\left[\frac{(1-\gamma) b+\left(\gamma-y_{a^{N}}\right) a}{1-y_{a^{N}}}, \frac{(1-\gamma) b+\left(\gamma-y_{a^{N}}\right) c}{1-y_{a^{N}}}\right]\right\}
$$

$$
=\left[\frac{(a+c)(1-\gamma)^{2}}{2}+\frac{(2 b-a-c)(1-\gamma)^{2}}{3\left(1-y_{a^{N}}\right)}\right]_{y_{a^{N}}}
$$$$
=\left[\frac{(a+c)\left(1-y_{a^{N}}\right)^{2}}{2}+\frac{(2 b-a-c)\left(1-y_{a^{N}}\right)^{a}}{3\left(1-y_{a^{N}}\right)}\right]
$$$$
=\frac{(3 a+3 c+4 b-2 a-2 c)\left(1-y_{a^{N}}\right)^{2}}{6}
$$$$
=\frac{(a+4 b+c)\left(1-y_{a^{N}}\right)^{2}}{6}
$$

$\int_{0}^{W_{a^{N}}} \alpha d \alpha=\left(\frac{\alpha^{2}}{2}\right)_{0}^{W_{a^{N}}}=\frac{w_{\alpha^{N}}{ }^{2}}{2}$

$$
\int_{0}^{1-u_{\alpha^{N}}} \beta d \beta=\left(\frac{\beta^{2}}{2}\right)_{0}^{1-u_{a^{N}}}=\frac{\left(1-u_{\alpha^{N}}\right)^{2}}{2}
$$

$$
\int_{0}^{1-y_{a^{N}}} y d y=\left(\frac{\gamma^{2}}{2}\right)_{0}^{1-y_{a^{N}}}=\frac{\left(1-y_{\left.a^{N}\right)^{2}}\right.}{2}
$$

Substitute the equation (1), (2), (3), (4), (5) and (6) in graded mean integration, we get

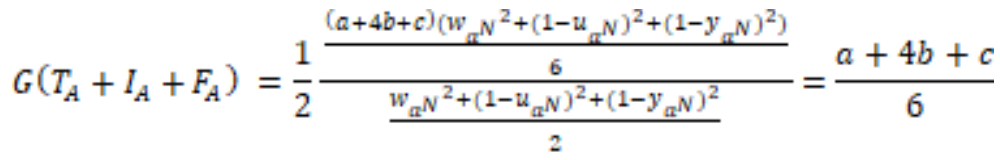




\section{Formulation of a Problem and Methodology}

\subsection{Single-Valued Neutrosophic Inventory Problem}

The single-valued neutrosophic inventory model of time independent profit maximizing neutrosophic costs can be thought of as a classic newsvendor problem releases where should a newsvendor buy the approximate number of newspapers for his corner newspaper shop such that he eventually reached the maximum expected profit.

Consider the item you can buy at the beginning of the period and after the end of the period, it is either used or sold at a price below the purchases price. Let,

$\mathrm{b}^{\mathbb{N}}=$ Neutrosophic unit price of purchased product (independent number of item purchased),

$\mathrm{a}^{\mathrm{N}}=$ Neutrosophic unit retail price of the products $\left(\mathrm{a}^{\mathrm{N}}<\mathrm{b}^{\mathrm{N}}\right)$,

$\mathrm{h}^{\mathbb{N}}=$ Neutrosophic holding cost per each item after the end of the period $\left(\mathrm{h}^{\mathrm{N}}<\mathrm{a}^{\mathbb{N}}\right)($ After

theend of the period a single price can be considered),

$\mathrm{s}^{\mathrm{N}}=$ Neutrosophic price of one product per defect,

and the demand $\Upsilon^{\mathbb{N}}$ as a neutrosophic random variable with an order pair is given as $\left\{\left(y_{1}^{\mathbb{N}}, p_{1}^{\mathbb{N}}\right),\left(y_{2}^{\mathbb{N}}, p_{2}^{\mathbb{N}}\right),\left(y_{2}^{\mathbb{N}}, p_{a}^{\mathbb{N}}\right), \ldots,\left(y_{n}^{N}, p_{n}^{\mathbb{N}}\right)\right\}$, If $y_{k}^{\mathbb{N}}$ products are purchased at the beginning of the period, then the neutrosophicprofit function $\mathrm{P}^{\mathrm{N}}$ is given by,

$P^{N}\left(y_{k}^{N}, Y^{N}\right)=\left\{\begin{array}{l}a^{N} y_{i}^{N}-b^{N} y_{k}^{N}-s^{N}\left(y_{k}^{N}-y_{i}^{N}\right), y_{i}^{N} \leq y_{k}^{N} \\ \left(a^{N}-b^{N}\right) y_{k}^{N}-s\left(y_{1}^{N}-y_{k}^{N}\right), y_{i}^{N} \geq y_{k}^{N}\end{array}\right.$

For some $\mathrm{i}=1$ ton.

As the neutrosophicdemand $\Upsilon^{\mathbb{N}}$ is a neutrosophic random variable, so its neutrosophicprofit function $\mathrm{P}^{\mathbb{N}}$ is also a neutrosophic random variable and obviously its total neutrosophic expected cost $\mathrm{EP}^{\mathrm{N}}$ becomes a unique neutrosophic number.Therefore the neutrosophic total expected profit $\left(\mathrm{E}^{\mathrm{N}} \mathrm{P}^{\mathrm{N}}\right)$ is determined by,

$E^{N} P^{N}\left(y_{k^{n}}^{N} \Upsilon^{N}\right)=\sum_{i=1}^{k}\left[a^{N} y_{i}^{N}-b^{N} y_{k}^{N}-s^{N}\left(y_{k}^{N}-y_{i}^{N}\right)\right] p_{i}^{N}+\sum_{i=k+1}^{n}\left[\left(a^{N}-b^{N}\right) y_{k}^{N}-s^{N}\left(y_{i}^{N}-y_{k}^{N}\right)\right] p_{i}^{N}$

\subsection{Mathematical Model with Neutrosophic Random Variable}

We consider the problem of stocks described above in a period when demand is seen as a neutrosophic random variable. The data are not accurate for neutrosophic probabilities so for simplicity, all data sets and probabilities are considered as triangular neutrosophic numbers $\left(\underline{y_{i}}, y_{i}, \overline{y_{i}}\right)^{N}$ and $\left(\underline{p_{i}}, p_{i}, \overline{p_{i}}\right)^{N}$ for $\mathrm{i}=1$ ton, respectively.

Now, the neutrosophic expected profit function $\mathrm{E}^{\mathrm{N}} \mathrm{P}^{\mathrm{N}}=(\underline{\mathrm{EP}}, \mathrm{EP}, \overline{\mathrm{EP}})^{\mathrm{N}}$ is given by

$$
\begin{gathered}
\mathrm{E}^{\mathrm{N}} \mathrm{P}^{\mathrm{N}}=\sum_{\mathrm{i}=1}^{\mathrm{k}}\left[\mathrm{a}^{\mathrm{N}} \mathrm{y}_{i}^{\mathrm{N}}-\mathrm{b}^{\mathrm{N}} \mathrm{y}_{\mathrm{k}}^{\mathrm{N}}-\mathrm{s}^{\mathrm{N}}\left(\mathrm{y}_{\mathrm{k}}^{\mathrm{N}}-\mathrm{y}_{\mathrm{i}}^{\mathrm{N}}\right)\right] \mathrm{p}_{\mathrm{i}}^{\mathrm{N}}+\sum_{\mathrm{i}=\mathrm{k}+1}^{\mathrm{n}}\left[\left(\mathrm{a}^{\mathrm{N}}-\mathrm{b}^{\mathrm{N}}\right) \mathrm{y}_{\mathrm{k}}^{\mathrm{N}}-\mathrm{s}^{\mathrm{N}}\left(\mathrm{y}_{\mathrm{i}}^{\mathrm{N}}-\mathrm{y}_{\mathrm{k}}^{\mathrm{N}}\right)\right] \mathrm{p}_{\mathrm{i}}^{\mathrm{N}} \\
=\sum_{\mathrm{i}=1}^{\mathrm{k}}\left[\left(\mathrm{a}^{\mathrm{N}}+\mathrm{s}^{\mathrm{N}}\right) \mathrm{y}_{\mathrm{i}}^{\mathrm{N}}-\left(\mathrm{b}^{\mathrm{N}}+\mathrm{s}^{\mathrm{N}}\right) \mathrm{y}_{\mathrm{k}}^{\mathrm{N}}\right] \mathrm{p}_{\mathrm{i}}^{\mathrm{N}}+\sum_{\mathrm{i}=\mathrm{k}+1}^{\mathrm{n}}\left[\left(\mathrm{a}^{\mathrm{N}}-\mathrm{b}^{\mathrm{N}}+\mathrm{s}^{\mathrm{N}}\right) \mathrm{y}_{\mathrm{k}}^{\mathrm{N}}-\mathrm{s}^{\mathrm{N}} \mathrm{y}_{\mathrm{i}}^{\mathrm{N}}\right] \mathrm{p}_{\mathrm{i}}^{\mathrm{N}}
\end{gathered}
$$

where, 


$$
\begin{aligned}
& (E P)^{N}=E^{N}\left[P_{\alpha+\beta+\gamma=\mathrm{a}}^{N}\right] \\
& =\sum_{i=1}^{k}\left[\left(a^{N}+s^{N}\right) y_{i}^{N}-\left(b^{N}+s^{N}\right) y_{k}^{N}\right] p_{i}^{N}+\sum_{i=k+1}^{n}\left[\left(a^{N}-b^{N}+s^{N}\right) y_{k}^{N}-s^{N} y_{i}^{N}\right] p_{i}^{N} \\
& =\sum_{i=1}^{k}\left[\left(a^{N}+s^{N}\right) y_{i}^{N} p_{i}^{N}-\left(b^{N}+s^{N}\right) y_{k}^{N} p_{i}^{N}\right]+\sum_{i=k+1}^{n}\left[\left(a^{N}-b^{N}+s^{N}\right) y_{k}^{N} p_{i}^{N}-s^{N} y_{i}^{N} p_{i}^{N}\right] \\
& \underline{(E P)}^{N}=E^{N}\left[P_{\alpha+\beta+Y=0^{-}}^{N}\right] \\
& =\sum_{i=1}^{k}\left[\left(a^{N}+s^{N}\right) \underline{y_{i}^{N} p_{i}^{N}}-\left(b^{N}+s^{N}\right) \underline{\left.y_{k}^{N} p_{i}^{N}\right]}+\sum_{i=k+1}^{n}\left[\left(a^{N}-b^{N}+s^{N}\right) \underline{y_{k}^{N} p_{i}^{N}}-s^{N} \underline{s^{N} y_{i}^{N} p_{i}^{N}}\right.\right. \\
& \overline{(E P)}^{N}=E^{N}\left[P_{\mathbb{E}+\beta+\gamma=a}^{N}\right] \\
& =\sum_{i=1}^{k}\left[\left(a^{N}+s^{N}\right) \overline{y_{i}^{N} p_{i}^{M}}-\left(b^{N}+s^{N}\right) \overline{y_{k}^{N} p_{i}^{M}}\right]+\sum_{i=k+1}^{n}\left[\left(a^{N}-b^{N}+s^{N}\right) \overline{y_{k}^{N} p_{i}^{M}}-s^{N} \overline{y_{i}^{M} p_{i}^{M}}\right]
\end{aligned}
$$

Here the $(\alpha+\beta+\gamma)$-level set of the neutrosophic number $\mathrm{E}^{\mathrm{N}} \mathrm{P}^{\mathrm{N}}$ are considered as follows,

$$
[E P]_{\alpha+\beta+Y}^{\mathbb{N}}=E^{\mathbb{N}}\left[P_{\alpha+\beta+Y}\right]^{\mathbb{N}}=\left[E^{\mathbb{N}}\left(P_{\alpha+\beta+Y}^{N}\right)^{-} E^{\mathbb{N}}\left(P_{\alpha+\beta+Y}^{N}\right)^{+}\right], 0 \leq \alpha+\beta+\gamma \leq 3
$$

and we get $\alpha+\beta+\gamma-$ cut interval with different neutrosophic number $\mathrm{E}^{\mathrm{N}} \mathrm{P}^{\mathrm{N}}$ for $\alpha+\beta+\gamma$

between 0 and 3.The membership function of this neutrosophic number $\mathrm{E}^{\mathrm{N}} \mathrm{P}^{\mathrm{N}}$ is defined by,

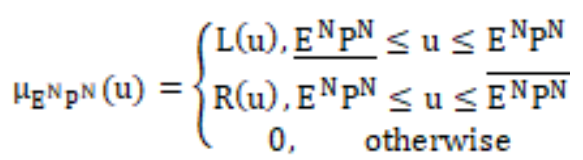

where $\mathrm{L}(\mathrm{u})$ is left continuous function from $\left[\mathrm{E}^{\mathbb{N}} \mathrm{P}^{\mathbb{N}}, \mathrm{E}^{\mathrm{N}} \mathrm{P}^{\mathrm{N}}\right]$ to $[0,1]$ and $\mathrm{R}(\mathrm{u})$ is the right continuous function from $\left[\mathrm{E}^{\mathrm{N}} \mathrm{P}^{\mathrm{N}}{ }_{\mathrm{E}^{\mathrm{E}}} \overline{\mathrm{PN}}\right]$ to $[0,1]$.Now, we use the method of indicating the amount of neutrosophic number that are summed based on the integral value.of graded mean $\alpha+\beta+\gamma-$ level, we find out a lost representative of the unique neutrosophic number $\mathrm{E}^{\mathrm{N}} \mathrm{P}^{\mathrm{N}}$ is

$$
\begin{aligned}
& \mathrm{G}\left(\mathrm{E}^{\mathbb{N}} \mathrm{P}^{\mathrm{N}}\right)=
\end{aligned}
$$

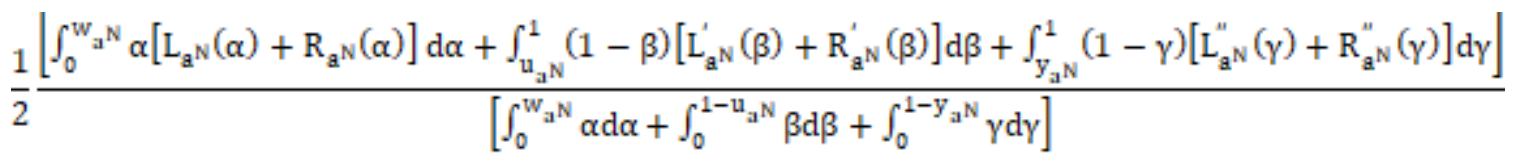

and we get,

$\mathrm{G}\left(\mathrm{E}^{\mathrm{N}} \mathrm{P}^{\mathrm{N}}\right)=\frac{\mathrm{E}^{\mathrm{N}} \mathrm{P}^{\mathrm{N}}+\mathrm{E}^{\mathrm{N}} \mathrm{P}^{\mathrm{N}}+\overline{\mathrm{E}^{\mathrm{N}} \mathrm{P}^{\mathrm{N}}}}{6}$

Moreover the neutrosophic optimal order quantity $\mathrm{y}_{\mathrm{k}}^{\mathrm{N}}$, we have

$\mathrm{G}\left(\mathrm{E}^{\mathrm{N}} \mathrm{P}^{\mathrm{N}}\left(\mathrm{y}_{\mathrm{k}^{\mathrm{N}}}^{\mathrm{N}} \Upsilon^{\mathrm{N}}\right)\right)>\mathrm{G}\left(\mathrm{E}^{\mathrm{N}} \mathrm{P}^{\mathrm{N}}\left(\mathrm{y}_{\mathrm{k}-1^{\mathrm{N}}}^{\mathrm{N}} \Upsilon^{\mathrm{N}}\right)\right)(7)$ 
$\operatorname{andG}\left(\mathrm{E}^{\mathrm{N}} \mathrm{P}^{\mathrm{N}}\left(\mathrm{y}_{\mathrm{k}}^{\mathrm{N}} \mathrm{Y}^{\mathrm{N}}\right)\right)>\mathrm{G}\left(\mathrm{E}^{\mathrm{N}} \mathrm{P}^{\mathrm{N}}\left(\mathrm{y}_{\mathrm{k}+1^{\mathrm{N}}}^{\mathrm{N}}, \mathrm{Y}^{\mathrm{N}}\right)\right)$

The result (7) and (8), are gives us the neutrosophic optimal order quantity $\mathrm{y}_{\mathrm{k}}^{\mathbb{N}}$, which satisfies as following,

lowerlimit $<\frac{a^{\mathbb{N}}-b^{\mathbb{N}}+s^{\mathbb{N}}}{a^{N}+b^{N}+s^{N}}<$ upperlimit,

where,

lowerlimit $=\frac{4\left(y_{k}^{N}-y_{k-1}^{N}\right) \sum_{i=1}^{k-1} p_{i}^{N}+\left(\underline{y_{k}^{N}}-\underline{y_{k-1}^{N}}\right) \sum_{i=1}^{k-1} \underline{p_{i}^{N}}+\left(\overline{y_{k}^{N}}-\overline{y_{k-1}^{N}}\right) \sum_{i=1}^{k-1} \overline{p_{i}^{N}}}{4\left(y_{k}^{N}-y_{k-1}^{N}\right) \sum_{i=1}^{n} p_{i}^{N}+\left(\underline{y_{k}^{N}}-\underline{y_{k-1}^{N}}\right) \sum_{i=1}^{n} \underline{p_{i}^{N}}+\left(\overline{y_{k}^{N}}-\overline{y_{k-1}^{N}}\right) \sum_{i=1}^{n} \overline{p_{i}^{N}}}$

and

upperlimit $=\frac{4\left(y_{k+1}^{N}-y_{k}^{N}\right) \sum_{i=1}^{k} p_{i}^{N}+\left(\underline{y_{k+1}^{N}}-\underline{y_{k-1}^{N}}\right) \sum_{i=1}^{k-1} \underline{p_{i}^{N}}+\left(\overline{y_{k+1}^{N}}-\overline{y_{k}^{N}}\right) \sum_{i=1}^{k} \overline{p_{i}^{N}}}{4\left(y_{k+1}^{N}-y_{k}^{N}\right) \sum_{i=1}^{n} p_{i}^{N}+\left(\underline{y_{k+1}^{N}}-\underline{y_{k-1}^{N}}\right) \sum_{i=1}^{n} \underline{p_{i}^{N}}+\left(\overline{y_{k+1}^{N}}-\overline{y_{k}^{N}}\right) \sum_{i=1}^{n} \overline{p_{i}^{N}}}$

Therefore, we conclude that the neutrosophic optimal order quantity can be determined over a single-valued neutrosophic inventory model when requirements are mentioned in a mixed environment, it is more accurate and less certain that it is more realistic for an inventory. Then if an inaccurate probability is specified for the search data set, we give a numerical example of how to get the neutrosophic optimal order quantity.

\section{Numerical Example}

To illustrate this model, suppose a newsvendor cannot pay in cash for a day if he needs more papers. Let the neutrosophic purchase price $\mathrm{b}^{\mathbb{N}}=5$, the neutrosophic price $\mathrm{a}^{\mathbb{N}}=8$, the neutrosophic holding $\cos \mathrm{h}^{\mathrm{N}}=3$, and the neutrosophic shortage price $\mathrm{s}^{\mathrm{N}}=6$. The daily neutrosophic demand for this section is unknown, but based on experience and previous sales dates, you can set neutrosophic probabilities for different search levels of neutrosophic demand. The neutrosophic demand and neutrosophic probability are given in the first table. Now, using our methodology you can find the neutrosophic optimal order quantity $y_{k}^{\mathbb{N}}$ (say) from second table.

Table 1:

\begin{tabular}{|c|l|}
\hline Neutrosophic demand & Neutrosophic probabilities \\
\hline$(23,24,25)(22,24,26)(21,24,27)$ & $(0.037,0.04,0.048)(0.032,0.04,0.053)(0.027,0.04,0.058)$ \\
\hline$(29,30,31)(28,30,32)(27,30,33)$ & $(0.134,0.16,0.150)(0.129,0.16,0.155)(0.124,0.16,0.160)$ \\
\hline$(35,36,37)(34,36,38)(33,36,39)$ & $(0.260,0.30,0.305)(0.255,0.30,0.310)(0.250,0.30,0.315)$ \\
\hline$(41,42,43)(40,42,44)(39,42,45)$ & $(0.169,0.20,0.205)(0.164,0.20,0.210)(0.159,0.20,0.215)$ \\
\hline$(47,48,49)(46,48,50)(45,48,51)$ & $(0.069,0.10,0.105)(0.064,0.10,0.110)(0.059,0.10,0.115)$ \\
\hline$(53,54,55)(52,54,56)(51,54,57)$ & $(0.070,0.10,0.104)(0.065,0.10,0.109)(0.060,0.10,0.114)$ \\
\hline$(59,60,61)(58,60,62)(57,60,63)$ & $(0.071,0.10,0.103)(0.066,0.10,0.108)(0.067,0.10,0.113)$ \\
\hline
\end{tabular}

Table 2: 


\begin{tabular}{|c|c|c|c|c|c|c|}
\hline$y_{k}^{N}$ & $(21,24,27)$ & $(27,30,33)$ & $(33,36,39)$ & $(39,42,45)$ & $(45,48,51)$ & $(51,54,57)$ \\
\hline $4\left(y_{k+1}^{N}-y_{k}^{N}\right) \sum_{i=1}^{k} p_{i}^{N}$ & 0.96 & 4.8 & 12 & 16.8 & 19.2 & 21.6 \\
\hline$\left(y_{k+1}^{N}-y_{k}^{N}\right) \sum_{i=1}^{k} p_{i}^{N}$ & 0.162 & 0.906 & 2.41 & 3.36 & 3.71 & 4.074 \\
\hline$\overline{\left(\overline{y_{k+1}^{N}}\right.}-\overline{y_{k+1}^{N}} \sum_{i=1}^{k} \overline{p_{i}^{N}}$ & 0.348 & 1.308 & 3.198 & 4.488 & 5.178 & 5.862 \\
\hline A & 1.47 & 7.014 & 17.608 & 24.68 & 28.118 & 31.554 \\
\hline $4\left(y_{k+1}^{N}-y_{k}^{N}\right) \sum_{i=1}^{M} p_{i}^{N}$ & 24 & 24 & 24 & 24 & 24 & 24 \\
\hline$\left(y_{k+1}^{N}-y_{k}^{N}\right) \sum_{i=1}^{M} p_{i}^{N}$ & 4.44 & 4.44 & 4.44 & 4.44 & 4.44 & 4.44 \\
\hline$\overline{\left(\overline{y_{k+1}^{N}}\right.}-\overline{y_{k+1}^{N}} \sum_{i=1}^{n} \overline{p_{i}^{N}}$ & 6.54 & 6.54 & 6.54 & 6.54 & 6.54 & 6.54 \\
\hline B & 34.98 & 34.98 & 34.98 & 34.98 & 34.98 & 34.98 \\
\hline$\frac{a^{N}-b^{N}+s^{N}}{a^{N}+b^{N}+s^{N}}$ & 0.5294 & 0.5294 & 0.5294 & 0.5294 & 0.5294 & 0.5294 \\
\hline$\frac{A}{B}$ & 0.0420 & 0.2005 & 0.5034 & 0.7055 & 0.8038 & 0.9021 \\
\hline
\end{tabular}

Let A and B be the numerator and denominator of the upper limit of (9) respectively. Then the neutrosophic optimal order quantity $\mathrm{y}_{\mathrm{k}}^{\mathrm{N}}$ is gives $(39,42,45)$, this means that for newsvendor it is better to buy about 42 newspapers in order to maximize the expected daily profit.

\section{Conclusion}

This paper, introduces the development of stochastic neutrosophic inventory models using neutrosophic random variables to get more realistic information, where fixed value is invalid. Here, a single-valued neutrosophic inventory models are discussed when there are inaccuracies and uncertainties in inventory system. This aggregation can be extended to other neutrosophic inventory models.

\section{Acknowledgement}

The article has been written with the joint financial support of RUSA-Phase 2.0 grant sanctioned vide letter No.F.24-51/2014-U, Policy (TN Multi-Gen), Dept. of Edn. Govt. of India, Dt. 09.10.2018, UGC-SAP (DRS-I) vide letter No.F.510/8/DRS-I/2016(SAP-I) Dt. 23.08 .2016 and DST (FST - level I) 657876570 vide letter No.SR/FIST/MS-I/2018-17 Dt. 20.12.2018.

\section{REFERENCES}

[1]Atanassov. KT (1999). Intuitionistic fuzzy sets. Pysica-Verlag A Springer-verlag company, New York. 
[2]Buffa . E.S. and SarinR.K.. (1987). Modern Production / Operations Management, John Wiley and Sons, Asia Pte Ltd.

[3]ChakraborthyD. (2002). Redefining chance-constrained programming in fuzzy environment, Fuzzy Sets and Systems 125,327-333.

[4]Deli I, Simsek I, Cagman N (2015). A multiple criteria group decision making methods on single valued trapezoidal neutrosophic numbers based on Einstein operations. The 4th international fuzzy systems symposium (FUZZYSS'15). YildizTechnical University, Istanbul, Turkey.

[5]Deli I, Subas y. A ranking method of single valued neutrosophic numbers and itsapplication to multi-attribute decision making problems, MuallimRifat Faculty of education, 7 Arahk University,79000 killis, Turkey.

[6]FengY., Hu L.and ShuH. (2001). The variance and covariance of fuzzy random variables and their applicatios, Fuzzy Sets and Systems 120, 487-497.

[7] HadleyG. and WhitenT.M. (1963). Analysis of Inventory Systems, Prentice-Hall, Englewood Cliffs, NJ.

[8]Ishii H.and Konno T. (1998). A stochastic inventory problem with fuzzy shortage cost, European J. Operational Research 106, 90-94.

[9]Kao C. and Hsu W.K.(2002). A single-period inventory model with fuzzy demand, Computers Math. Applic. 43 (6/7), 841-848.

[10]Kim Y.K and GhilB.M. (1997).Integrals of fuzzy number valued functions, Fuzzy Sets and Systems 86, 213222.

[11]KwakernaakH. (1978). Fuzzy random variables: Definition and theorems, Information sciences15, 1-29.

[12]LiL., KabadiS.N. and Nair K.P.K. (2002). Fuzzy models for single-period inventory problem, Fuzzy Sets and System 132, 273-289.

[13]Lopez-Diaz . M.and Gil M.A. (1998). The ג-average value of the expected value of a fuzzy random variable, Fuzzy random variable Fuzzy Sets and Systems 99, 347-391.

[14]Puri M.L. andRalescuD.A. (1986).Fuzzy random variables, J. Mathematical Analysis and Applications 114, 409-422.

[15]Smarandache F. (1998). A unifying field in logics neutrosophy: neutrosophic probability, set and logic. American Research Press, Rehoboth.

[16]Subas Y. (2015).Neutrosophic numbers and their application to Multi-attribute decision making problems (in Turkish) (Masters Thesis, Kilis 7 Arahk university, Graduate School of Natural and Applied Science)

[17]Wang H, Smarandache F, Y, Zhang Q (2010). Single valued neutrosophic sets. MultispaceMultistructure 4:410413.

[18]Zadeh L.A. (1965). Fuzzy Sets. Information Control 8:338-353. 